\section{Beta-Blocker bei Asthma und COPD - ein therapeutisches Dilemma?}

\author{
Beta-Blocker bei Asthma und COPD - ein therapeutisches \\ Dilemma?
}

Die Substanzklasse der Beta-Rezeptorenblocker hat eine besondere Bedeutung in der Pharmakotherapie von kardiovaskulären Erkrankungen wie der arteriellen Hypertonie, der koronaren Herzkrankheit, Herzrhythmusstörungen oder der chronischen Herzinsuffizienz gewonnen. Der Wirkungsmechanismus der Beta-Blocker beruht darauf, die Bindung der Katecholamine an Beta-Rezeptoren zu hemmen. Die einzelnen Beta-Blocker entfalten prinzipiell ähnliche Wirkungen (Tab.1), sie unterscheiden sich jedoch in einigen pharmakodynamischen und pharmakologischen Eigenschaften. Grundsätzlich lassen sich unselektive Blocker (Propanolol, Nadolol, Penbutolol, Carvedilol u.a.) von Beta-1-selektiven Rezeptoren-Blockern (Atenolol, Bisoprolol, Metoprolol u.a.) unterscheiden (Tab. 2). Trotz relativer Beta-1-Selektivität ist diese nicht strikt vorhanden - ein Aspekt, der besondere Bedeutung bei Lungenkrankheiten hat. Ein weiteres Unterscheidungsmerkmal ist die intrinsisch-sympathomimetische Aktivität einiger Beta-Blocker (Oxprenolol, Pindolol u.a.), da für sie eine Reduktion der Mortalität bei koronarer Herzkrankheit nicht nachgewiesen werden konnte [7].

Die Hauptindikationen für den Einsatz von Beta-Rezeptorenblockern sind in Tab. 3 dargestellt. Angesichts der positiven Effekte der Beta-Blocker auf die Morbidität und vor allem auch auf die Mortalität bei kardiovaskulären Erkrankungen einerseits und der bronchokonstriktischen Wirkung bei obstruktiven Atemwegserkrankungen andererseits ist der Einsatz dieser Substanzgruppe für Patienten mit Asthma und COPD kritisch zu diskutieren. Sicherlich kann eine Verschlechterung des Asthma bronchiale oder der chronisch obstruktiven Bronchitis durch den Verzicht auf Beta-Blocker bei diesen Patienten verhindert werden, andererseits wird ihnen auch der Nutzen, z.B. infolge einer Reduktion der Mortalität um $40 \%$ nach Herzinfarkt [13], vorenthalten.

Insofern ist die Indikation zum Einsatz von Beta-Blockern bei Patienten mit Asthma bronchiale und COPD unter Berücksichtigung der Art der Erkrankung, des Schweregrades, der potenziellen Indikationen für den Einsatz von Beta-Blockern und des zu erwartenden Nutzens sowie der Möglichkeit medikamentöser Alternativen kritisch abzuwägen.

Pneumologie 2001; 55: 53-56

(c) Georg Thieme Verlag Stuttgart · New York ISSN 0934-8387
H. Worth

Medizinische Klinik I, Klinikum Fürth

Tab. 1 Pharmakodynamische Wirkungen unter Beta-Blockade

Verminderung der Noradrenalin-Freisetzung

Verminderung des peripheren Gefäßwiderstandes

Resetting des Barorezeptorenreflexes

Verminderung der Reaktionen auf Katecholamine unter Belastung Verminderung des venösen Rückflusses und des Plasma-Volumens Inhibition des Renins

Verminderung des Schlagvolumens, der Herzfrequenz und des myokardialen $\mathrm{O}_{2}$-Verbrauchs

zentral-venöse Effekte

modifiziert nach [9]

Tab. 2 Pharmakodynamische Unterschiede von Beta-RezeptorenBlockern (Auswahl)

\begin{tabular}{|c|c|c|c|c|}
\hline Substanz & \multicolumn{2}{|c|}{$\begin{array}{l}\text { Beta-Rezeptor- } \\
\text { Selektivität }\end{array}$} & $\begin{array}{l}\text { Vasodilata- } \\
\text { tion }\end{array}$ & $\begin{array}{l}\text { intrinsische } \\
\text { sympatho- } \\
\text { mimetische }\end{array}$ \\
\hline \multicolumn{5}{|l|}{ Aktivität } \\
\hline Atenolol & $x$ & & & \\
\hline Bisoprolol & $x$ & & & \\
\hline Carvedilol & $x$ & $x$ & $x$ & \\
\hline Celiprolol & $x$ & & $x$ & + \\
\hline Esmolol & $x$ & & & \\
\hline Oxprenolol & $x$ & $x$ & & ++ \\
\hline Pindolol & $x$ & $x$ & & +++ \\
\hline Sotalol & $x$ & $x$ & & \\
\hline
\end{tabular}

modifiziert nach [9]

Tab. 3 Wesentliche Indikationen für den Einsatz von Beta-Blockern

arterielle Hypertonie

koronare Herzkrankheit, insbesondere Zustand nach Myokardinfarkt Herzrhythmusstörungen supraventrikulärer und ventrikulärer Genese chronische Herzinsuffizienz

hyperkinetisches Herzsyndrom

Glaukom

Hyperthyreose

Migräne

Phäochromozytom

essentieller Tremor

portale Hypertension 


\section{Unerwünschte Effekte von Beta-Blockern bei obstruktiven} Atemwegserkrankungen

Die Verschlechterung eines vorbestehenden Asthma bronchiale unter Beta-Blockern wurde bereits kurz nach Einführung dieser Substanzklasse in die klinische Praxis beobachtet [22]. Obwohl diese Eigenschaft der Beta-Blocker seit langem bekannt ist, wird immer wieder über lebensbedrohliche Zwischenfälle bei Asthmatikern, auch bei Patienten mit leichtem Schweregrad der Erkrankung, unter dem Einsatz von Beta-Blockern berichtet [14]. Die zur Bronchokonstriktion führende Dosis des Beta-Blockers kann insbesondere bei Asthmatikern in Abhängigkeit von den Begleitumständen (Infekte, Allergenexposition) niedrig sein. So wird selbst durch den Einsatz beta-blocker-haltiger Augentropfen in der Behandlung des Glaukoms über schwere Asthma-Anfälle bis hin zu Todesfällen berichtet $[8,11,28]$.

Mehr als 90\% der Asthmatiker zeigen nach Gabe des unselektiven Beta-Blockers Propanolol eine Bronchokonstriktion [35]. Das Ausmaß der Bronchokonstriktion bei Applikation eines Beta-Blockers kann hierbei nicht sicher vorhergesagt werden und korreliert offensichtlich nicht mit dem Schweregrad einer bronchialen Hyperreaktivität. Die Sensitivität gegenüber Beta-Blockern ist ausgeprägter bei Patienten mit gutem Ansprechen auf Beta-2-Sympathomimetika im BronchospasmolyseTest [16]. Dies wird gestützt durch die Beobachtungen bei Patienten mit chronisch obstruktiver Bronchitis und/oder Lungenemphysem, bei denen nach Gabe von Beta-Blockern seltener eine Verschlechterung der Lungenfunktion auftritt $[20,24]$. Ferner ist die Verschlechterung der Lungenfunktion meist weniger ausgeprägt als bei Patienten mit Asthma bronchiale ( $\mathrm{cf}[30])$.

Beta-1-selektive Blocker führen seltener und in geringerem Ausmaß als nicht-selektive Beta-Blocker zur Bronchialobstruktion bei obstruktiven Lungenkrankheiten [10,12,15,19,27,34]. Außerdem ist die unter Beta-1-selektiven Beta-Blockern auftretende Bronchialobstruktion durch Inhalation von Beta-2Sympathomimetika reversibel [5]. Nicht-selektive Beta-Blocker mit intrinsischer sympathomimetischer Aktivität wie Pindolol verursachen weniger häufig eine Bronchokonstriktion als Substanzen ohne intrinsische Aktivität [4]. Die durch nicht-selektive Beta-Blocker verursachte Bronchokonstriktion kann allerdings nicht durch Beta-2-Agonisten beseitigt werden. Beta-Blocker mit intrinsischer Aktivität haben sich in der Behandlung nach Herzinfarkt und bei chronischer Herzinsuffizienz nicht bewährt, so dass ihr Einsatz bei Patienten mit obstruktiven Atemwegserkrankungen angesichts gleichwertiger Alternativpräparate nicht indiziert ist.

\section{Mechanismen der durch Beta-Blocker hervorgerufenen Bronchokonstriktion}

Die Mechanismen, die zur beta-blockerinduzierten Obstruktion bei Asthmakranken führen, sind bisher nicht genau geklärt. Ein wahrscheinlicher Mechanismus für die betablockerinduzierte Bronchokonstriktion bei Asthmatikern wird in einer Zunahme neuraler bronchokonstriktorischer Aktivität gesehen. Über Beta-2-Rezeptoren und ihre Aktivierung durch Adrenalin wird die Acetylcholin-Freisetzung an cholinergen Nerven in den menschlichen Atemwegen moduliert und der cholinerge Tonus gedämpft. Bei der Blockade dieser Rezepto- ren steigt die Acetylcholin-Freisetzung, wodurch über die Stimulation präjunktionaler $\mathrm{M}_{2}$-Autorezeptoren bei Lungengesunden eine weitere Freisetzung von Acetylcholin und damit eine Steigerung des Tonus der Atemwege verhindert werden $[1,3,25]$. Durch einen Defekt im Bereich der $\mathrm{M}_{2}$ Rezeptoren in den Atemwegen asthmakranker Patienten kann die durch Beta-blocker-induzierte gesteigerte Freisetzung von Acetylcholin im Gegensatz zur Situation bei Lungengesunden nicht kompensiert werden. Infolge der gesteigerten Acetylcholin-Freisetzung werden mehr $\mathrm{M}_{3}$-Rezeptoren der glatten Atemwegsmuskulatur erreicht.

Zusätzlich ist die bronchokonstriktorische Reaktion auf Acetylcholin bei Asthmatikern verstärkt und kann zur Bronchokonstriktion nach Beta-Blocker-Einnahme beitragen. Da Defekte an den $\mathrm{M}_{2}$-Rezeptoren auch bei Patienten mit leichtem Asthma beobachtet werden, ist die auch bei leichtgradigem Asthma beobachtete Verschlechterung unter Beta-Blockergabe erklärbar $[2,23]$. Diese Theorie wird auch durch die Tatsache gestützt, dass die beta-blocker-induzierte Bronchokonstriktion bei Asthmatikern durch inhalative Anticholinergika gebessert werden kann [17].

Bei einigen Mutanten von Beta-2-Rezeptoren wurden Aktivität und Aktivierung von G-Proteinen festgestellt, auch in Abwesenheit der Rezeptorbesetzung durch einen Agonisten [21]. In dieser Situation wirken Beta-Blocker als inverse Agonisten, wobei verschiedene Beta-Blocker unabhängig vom Ausmaß ihrer beta-blockierenden Potenz unterschiedlich starke inverse Agonisten sind. So wirkt Propanolol als starker, Pindolol hingegen als schwacher inverser Agonist. Auch dies mag zur Erklärung der unterschiedlichen Ausprägung der Bronchokonstriktion verschiedener Beta-Blocker bei Asthmatikern beitragen.

Bei Patienten mit schwerem Asthma wird als Mechanismus der Bronchokonstriktion durch Beta-Blocker auch die fehlende Inhibition der Tachykinin-Freisetzung von sensorischen Nerven der Atemwege diskutiert [18,31]. Die Gabe von BetaBlockern führt in dieser Situation zu einer gesteigerten Freisetzung der Neuropeptide, die die Atemwegsentzündung und die Bronchokonstriktion verstärken.

\section{Konsequenzen für den Einsatz von Beta-Blockern bei Patienten mit obstruktiven Atemwegserkrankungen}

Aus den Resultaten der angegebenen Studien lässt sich eine Verschlechterung der Lungenfunktion infolge der betablockerinduzierten Bronchokonstriktion bei Patienten mit Asthma bronchiale oder COPD ableiten. Das Risiko einer bedrohlichen Bronchialobstruktion unter Einsatz von BetaBlockern ist bei Asthmatikern deutlich höher als bei Patienten mit chronisch obstruktiver Bronchitis und/oder Lungenemphysem.

Bei Patienten mit Asthma bronchiale kann, wenn auch mit etwas geringerer Wahrscheinlichkeit, auch bei leichten und mittleren Schweregraden bei Applikation von Beta-Blockern eine schwere Obstruktion auftreten. Das Ausmaß der Bronchokonstriktion unter Einnahme eines Beta-Blockers kann anhand einer einmaligen Gabe einer Einzeldosis nicht exakt vorhergesagt werden, da die zu Asthma-Anfällen führenden 
Umstände eine beta-blocker-induzierte Obstruktion verstärken können.

Vor Einsatz eines Beta-Blockers ist beim Patienten zu prüfen, ob eine alternative Medikation ohne ungünstige Auswirkung auf die Lungenfunktion des Patienten zur Verfügung steht. Für die meisten Indikationen, insbesondere die arterielle Hypertonie und das Glaukom sowie die Herzinsuffizienz, steht diese in Form von Thiaziddiuretika, Kalziumantagonisten, ACEHemmern, Hydralazin, Clonidin, AT I-Antagonisten und Alphamethyldopa zur Verfügung. Auch bei den meisten Formen der ventrikulären und supraventrikulären Rhythmusstörungen kann mit Hilfe von Antiarrhythmika ohne beta-blockierende Eigenschaften auf die Gabe des Beta-Blockers verzichtet werden, vielleicht mit Ausnahme des seltenen, bei Asthmatikern allerdings etwas häufiger beobachteten Syndroms mit verlängerter QT-Dauer [26].

Angesichts der Reduktion der Mortalität nach Herzinfarkt durch den Einsatz von Beta-Blockern auch für Patienten mit obstruktiven Atemwegserkrankungen [13] ist für diese Indikation der Einsatz von Beta-Blockern bei Patienten mit Asthma bzw. COPD zu diskutieren. Bei Rauchern findet sich eine höhere Ko-Morbidität von koronarer Herzkrankheit und COPD. In dieser Patientengruppe stellt sich die Indikation zur Beta-Blockertherapie bei Zustand nach Herzinfarkt häufiger als beim Asthma. Da bei COPD-Patienten häufig eine irreversible Obstruktion und allenfalls ein geringes Ansprechen der Obstruktion auf Beta-2-Sympathomimetika beobachtet wird, kann der Beta-Blocker mit geringerem Risiko als bei Asthmatikern eingesetzt werden. In dieser Gruppe sollte der Einsatz des Beta-Blockers nach Herzinfarkt unter sorgfältiger Beobachtung des Patienten und Wahl einer niedrigen Initialdosis keine Kontraindikation darstellen. Vorzuziehen sind Beta-1selektive Substanzen.

Ferner erscheint für Patienten mit COPD und erhöhtem kardiovaskulärem Risiko perioperativ der Einsatz von BetaBlockern bei größeren operativen Eingriffen zur Reduktion einer myokardialen Ischämie, des Auftretens von Herzinfarkten in der unmittelbar postoperativen Phase bzw. zur Verhinderung und Reduktion von Arrhythmien indiziert (cf. [33]).

Vorsicht ist geboten bei Patienten mit asthmatischer Bronchitis, d.h. der Gruppe von COPD-Patienten, die eine höhere Reversibilität der Obstruktion und eine bronchiale Hyperreaktivität aufweisen. Ein erhöhtes Risiko bzgl. einer Verschlechterung der Lungenfunktion unter Beta-Blockern ist ferner bei älteren Patienten vorhanden, bei denen eine Unterscheidung zwischen Asthma bronchiale und COPD infolge der wenig reversiblen Obstruktion bei lange bestehendem Asthma nicht mehr gegeben ist [30].

Bei Patienten mit Asthma bronchiale sollten Beta-Blocker angesichts des gegenüber COPD-Patienten höheren Risikos einer unvorhersehbaren schweren Atemwegsobstruktion prinzipiell nicht eingesetzt werden. Eine absolute Kontraindikation besteht für Patienten mit schwerem Asthma sowie im Asthma-Anfall. Sollte aus vitaler Indikation, z.B. einer nicht anders beherrschbaren lebensbedrohlichen Herzrhythmusstörung, der Einsatz eines Beta-Blockers notwendig sein, ist ein kardioselektiver Beta-Blocker zu empfehlen, der in möglichst niedriger Anfangsdosis unter sorgfältiger Beobach- tung des Patienten eingesetzt wird. Die Therapie sollte unter gleichzeitiger Medikation mit Beta-2-Sympathomimetika und, sofern keine Kontraindikation besteht, auch von Anticholinergika erfolgen [29]. Bevor der Einsatz von Beta-Rezeptorenblockern beim Asthmatiker nach Herzinfarkt $[6,29,32]$ empfohlen werden kann, ist zu prüfen, ob bei Patienten mit klar definierten Schweregraden der Erkrankung Beta-Blocker auch in niedriger Dosierung zu einer Reduktion der Mortalität führen, wie dies in der retrospektiven Studie von Gottlieb et al. [12] für Patienten mit Asthma und COPD ohne klare Definition des Asthma-Schweregrades und der Charakterisierung der Patienten, die mit einer Bronchokonstriktion auf den Beta-Blocker reagierten, angegeben wurde [31].

\section{Fazit}

Beta-Blocker können bei Patienten mit Asthma bronchiale und COPD eine Bronchialobstruktion auslösen. Hiervon sind Asthmatiker häufiger und in stärkerem Ausmaß betroffen als Patienten mit COPD. Zur Vermeidung einer durch Beta-Blocker induzierten Bronchokonstriktion sollten bei obstruktiven Atemwegserkrankungen medikamentöse Alternativen bevorzugt werden, die für die meisten Indikationen, insbesondere die arterielle Hypertonie, das Glaukom, die Herzinsuffizienz und auch die supraventrikulären und ventrikulären Rhythmusstörungen zur Verfügung stehen. Nach Herzinfarkt und perioperativ bei erhöhtem kardiovaskulären Risiko sind Beta1-selektive Substanzen bei Patienten mit COPD indiziert, da der Nutzen mit Reduktion der Morbidität und der Mortalität nach Infarkt die potentiellen Risiken bei sorgfältiger Beobachtung des Patienten und geringer Initialdosis des Beta-Blockers überwiegt. Bei Patienten mit Asthma bronchiale sollten BetaBlocker angesichts des gegenüber COPD-Patienten höheren Risikos einer unvorhersehbaren schweren Atemwegsobstruktion prinzipiell nicht eingesetzt werden.

\section{Literatur}

${ }^{1}$ Aizawa $\mathrm{H}$, Inoue H, Miyazaki N, Ikeda T, Shigematsu N, Itou Y. Effects of procaterol, a beta-2-adrenozeptor stimulant, on neuroeffector transmission in human bronchial tissue. Respiration 1991; 58: $163-166$

2 Ayala LE, Ahmed T. Is there a loss of protective muscarinic rezeptor mechanism in asthma? Chest 1991; 96: 1285-1290

${ }^{3}$ Barnes PJ. Muscarinic receptor subbtypes: implications for lung disease. Thorax 1989; 44: 161 - 167

${ }^{4}$ Benson MK, Berrill WT, Gruikshank JM, Sterling GS. A comparison of four beta-adrenoceptor antagonists in patients with asthma. Br J Clin Pharmacol 1978; 5: 415-419

${ }^{5}$ Carstairs JR, Nimmo AJ, Barnes PJ. Autoradiographic visualization of betaadrenoceptor subtypes in human lung. Am Rev Respir Dis 1985; $132: 541-547$

${ }^{6}$ Chafin CC, Soberman JE, Demirkan K, Self T. Beta-blockers after myocardial infarction: do benefits ever outweigh risks in asthma? Cardiology 1999; 92: 99-105

${ }^{7}$ Christ M, Wehling M. Betablocking agents in patients with coronary artery disease and myocardial infarction. From hypertension to heart failure. In: Boehm M, Zehender M (Hrsg). Heidelberg: Springer, 1998: 90-129

${ }^{8}$ Daun TL, Gerber MJ, Shen AS, Fernandez E, Iserman MD, Cherniak RM. The effect of topic ophthalmic instillation of timolol and betaxolol on lung function in asthmatic subjects. Am Rev Respir Dis 1986; 133: 264-268 
${ }^{9}$ Feuring M, Schmidt BMW, Wehling M. Beta-Blocker. Stellenwert bei kardiovaskulären Erkrankungen. Internist 1999; 40: 680685

${ }^{10}$ Fogari R, Zoppi A, Teltamanti F, Poletti L, Rizzandi G, Fiocchi G. Comparative effects of celiprolol, propanolol, oxprenolol, and atenolol on respiratory function in hypertensive patients with chronic obstructive lung disease. Cardiovasc Drugs Ther 1990; 4: $1145-1150$

${ }^{11}$ Fraunfelder FT, Baker AF. Respiratory effects of timolol. N Engl J Med 1984; 311: 1441

12 Gold MR, Dec GW, Cocca-Spofford D, Thompson BT. Esmolol and ventilatory function in cardiac patients with COPD. Chest 1991; 100: $1215-1218$

${ }^{13}$ Gottlieb SS, McCarter RJ, Vogel RA. Effect of beta-blockade on mortality among high-risk and low risk patients after myocardial infarction. N Engl J Med 1998; 339: 489-497

${ }^{14}$ Graft DF, Fowles J, McCoy CE, Lager RA. Detection of beta-blocker in people with asthma. Ann Allergy 1992; 69: 449-453

${ }^{15}$ Greefhorst AM, van Herwaarden CLA. Comparative study of the ventilatory effects of three beta-1-selective blocking drugs in asthmatic patients. Eur J Clin Pharmacol 1981; 20: 417-421

${ }^{16}$ van Herwaarden CLA. Betaadrenoceptor blockade and pulmonary functions in patients suffering from chronic obstructive lung disease. J Cardiovasc Pharmacol 1983; 5: S46-S50

${ }^{17}$ Ind PW, Dixon CMS, Fuller RW, Barnes PJ. Anticholinergic blockade of beta-blocker induced bronchoconstriction. Am Rev Respir Dis 1989; 139: 1390-1394

18 Kamikawa Y, Shimo Y. Inhibitory effects of catecholamines on cholinergically and noncholinergically mediated contractions of guinea pig isolated bronchial muscle. J Pharm Pharmacol 1990; 42: $131-134$

${ }^{19}$ Lammers JWJ, Folgering HTM, van Herwaarden CLA. Ventilatory effects of beta-1-receptor selective blockade with bisoprolol and metoprolol in asthmatic patients. Eur J Clin Pharmacol 1984; 27: $141-145$

${ }^{20}$ Lammers JWJ, Folgering HTM, van Herwaarden CLA. Ventilatory effects of long-term treatment with pindolol and metoprolol in hypertensive patients with chronic obstructive lung disease. $\mathrm{Br} \mathrm{J}$ Clin Pharmacol 1985; 20: 205-210

${ }^{21}$ Leff P. Inverse agonism: theory and practise. Trends Pharmacol Sci 1995; 16: 256-259

22 Mc Neill RS. Effect of beta-adrenergic blocking agent, propanolol, on asthmatics. Lancet 1964: $1101-1102$

${ }^{23}$ Minette PAH, Lammers J, Dixon CMS, Mc Cusker MT, Barnes PJ. A muscarinic agonist inhibits reflex broncoconstriction in normal but not in asthmatic subjects. J Appl Physiol 1989; 67: 2461 2465

${ }^{24}$ Perks W, Chatterjee S, Croxson R, Criukshank J. Comparison of atenolol and oxprenolol in patients with angina or hypertension and coexistent chronic airways obstruction. Br J Clin Pharmacol 1985; 20: 205-210

${ }^{25}$ Rhoden KJ, Meldrum LA, Barnes PJ. Inhibition of cholinergic neurotransmission in human airways by beta-2-adrenoceptors. J Appl Physiol 1988; 65: 700 - 705

${ }^{26}$ Rosero SZ, Zareba W, Moss AJ, Robinson JL, Raef HAA, Locati EH, Benhorin J, Andrews ML. Asthma and the risk of cardiac events in the long QT syndrome. Am J Cardiol 1999; 84: 1406-1411

${ }^{27}$ Ruffin RE, Frith MB, Anderton RL, Kumana CR, Newhouse MT, Hargraeve FE. Selectivity of beta-andrenoceptor antagonist drugs assessed by histamine bronchial provocation. Clin Pharmacol Ther 1979; 25: 536-540

${ }^{28}$ Shoene RB. Timolol-induced bronchospasm in asthmatic bronchitis. JAMA 1981; 245: 1460

${ }^{29}$ Tafreshi MJ, Weinacker AB. Beta-adrenergic-blocking agents in bronchospastic diseases: a therapeutic dilemma. Pharmacotherapy 1999; 19: $974-978$
${ }^{30}$ Tattersfield A. Respiratory function in the elderly and the effects of beta-bockade. Cardiovasc Drugs Ther 1990; 4: 1229-1232

${ }^{31}$ Verleden GM, Belvisi MG, Rabe KF, Miura M, Barnes PJ. Beta-2Adrenoceptors inhibit NANC neural bronchoconstrictor responses in vitro. J Appl Physiol 1993; 74: 1195-1199

${ }^{32}$ White CM. Prevention of suboptimal beta-blocker treatment in patients with myocardial infarction. Ann Pharmacother 1999; 33: $1063-1072$

${ }^{33}$ Wiener-Kronish JP, Albert RK. Preoperative Evaluation. In: Murray JF, Nadel JA, Mason RJ, Boushey HA (Hrsg) Textbook of Respiratory Medicine. 3rd edition. Philadelphia: Saunders, 2000; 29: $883-894$

${ }^{34}$ Wilcox PE, Ahmad D, Darke A, Parsons J, Carruthers SG. Respiratory and cardiac effects of metoprolol and bevantolol in patients with asthma. Clin Pharmacol Ther 1986; 39: 29-34

${ }^{35}$ Woolcock AJ, Anderson SA, Reat JK, Du Toit JI, Zhang YG, Smith CM, Salome CM. Characteristics of bronchial hyperresponsiveness in chronic obstructive pulmonary disease and in asthma. Am Rev Respir Dis 1991; 143: 1438-1443

\section{Prof. Dr. H. Worth}

Medizinische Klinik I

Klinikum Fürth

Jakob-Henle-Str. 1

90766 Fürth

E-mail: med1@klinikum-fuerth.de 